\title{
Accurate determination of the specific absorption rate in superparamagnetic nanoparticles under non-adiabatic conditions
}

\author{
F. J. Teran, ${ }^{1}$ C. Casado, ${ }^{1}$ N. Mikuszeit, ${ }^{1,2}$ G. Salas,,${ }^{1,3}$ A. Bollero, ${ }^{1}$ M. P. Morales,${ }^{3}$ \\ J. Camarero, ${ }^{1,2}$ and R. Miranda ${ }^{1,2}$ \\ ${ }^{1}$ Instituto Madrileño de Estudios Avanzados en Nanociencia, Ciudad Universitaria de Cantoblanco, \\ 28049 Madrid, Spain \\ ${ }^{2}$ Dpto. de Física de la Materia Condensada and Instituto Nicolás Cabrera, Universidad Autónoma de Madrid, \\ 28049 Madrid, Spain \\ ${ }^{3}$ Instituto de Ciencia de Materiales de Madrid, Consejo Superior de Investigaciones Científicas, \\ Calle Sor Juana Ines de la Cruz 3, 28049 Madrid, Spain
}

(Received 11 May 2012; accepted 24 July 2012; published online 8 August 2012)

\begin{abstract}
We report on a general description of non-adiabatic calorimetry measurements for improving the accuracy on the determination of the specific absorption rate of superparamagnetic nanoparticles subjected to alternating magnetic fields. We perform experiments on reduced volumes of iron oxide nanoparticles dispersed in aqueous media under different thermal equilibrium conditions. We introduce a simple model, which considers linear thermal losses to precisely reproduce the complete time evolution of temperature. The control and the quantification of heat losses lead to higher accuracy for determining the specific absorption rate in superparamagnetic nanoparticles. (C) 2012 American Institute of Physics. [http://dx.doi.org/10.1063/1.4742918]
\end{abstract}

Superparamagnetic iron oxide nanoparticles (SPION) are of great interest for biomedical applications because of their suitable magnetic, colloidal, and structural properties. Different chemical synthesis methods allow to produce SPION with distinct biocompatible coatings, ${ }^{1}$ controlled aggregate size, and high colloidal stability. ${ }^{2}$ SPION have shown potential applications as contrast agents, ${ }^{3}$ drug delivery nanocarriers, ${ }^{4}$ and local heating inductors. ${ }^{5}$ For the latter applications, the iron mass-normalized specific absorption rate $(\mathrm{SAR})^{5}$ is the commonly used physical magnitude for evaluating the calorific power of SPION subjected to alternating magnetic fields $\left(H_{\mathrm{AC}}\right)$. SAR is determined by standard calorimetric methods ${ }^{6}$ according to the expression $\mathrm{SAR}=C / m \mathrm{~d} T /\left.\mathrm{d} t\right|_{\mathrm{t}=0}$, where $C$ is the specific heat capacity of the sample, $m$ is the iron mass of magnetic material in solution, and $\mathrm{d} T /\left.\mathrm{d} t\right|_{\mathrm{t}=0}$ is the slope of the temperature increase at initial times after applying $H_{\mathrm{AC}}$. An accurate determination of SAR is hence essential for quantifying the magnetic heating power of SPION for their application as intracellular hyperthermia generators.

SPION SAR values tightly depend on nanoparticle parameters such as $\operatorname{size}^{7,8}$ or concentration, ${ }^{9,11}$ or sample volume, ${ }^{12}$ nanoparticle interactions ${ }^{10}$ and alternating magnetic field parameters such as frequency, amplitude, ${ }^{7,13}$ or field inhomogeneities. ${ }^{12}$ Adiabatic or non-adiabatic experimental conditions have also a strong influence in the SAR quantification. ${ }^{14}$ Adiabatic conditions provide more accurate SAR values than those obtained under non-adiabatic ones. The latter always requires the performance of calorimetry measurements under thermal equilibrium conditions in order to extract the right value of $\mathrm{d} T /\left.\mathrm{d} t\right|_{\mathrm{t}=0}$ necessary for SAR determination. In this manner, the $\mathrm{d} T /\left.\mathrm{d} t\right|_{\mathrm{t}=0}$ value will be scarcely influenced by thermal losses of SPION into the surrounding via radiative, convection, and conduction mechanisms. ${ }^{15}$ However, non-adiabatic conditions provide a more realistic scenario for applying the intracelullar magnetic heating power of SPION in both in vitro ${ }^{16}$ and in vivo ${ }^{17}$ studies. Thus, the control and the quantification of SPION thermal losses with the surrounding are required to determine SAR values with higher accuracy.

The purpose of this letter is to provide a general description of non-adiabatic calorimetry measurements performed on reduced volumes of SPION (tens of $\mu \mathrm{l}$ ) subjected to $H_{\mathrm{AC}}$ in order to quantify more accurately their SAR value. We have carried out experiments at different equilibrium temperatures $\left(T_{\text {eq }}\right)$ in the range from $10^{\circ} \mathrm{C}$ to $30^{\circ} \mathrm{C}$ and applying $H_{\mathrm{AC}}$ under different equilibrium conditions. We propose a simple model to reproduce the time variation of SPION temperature when $H_{\mathrm{AC}}$ is turned on and off. The model is based on the linear heat losses of SPION into the surrounding. Our experimental findings point out the advantage of performing calorimetry measurements on reduced SPION volumes in order to determine more accurate SAR values.

$\mathrm{Fe}_{3} \mathrm{O}_{4}$ SPION were synthesized by modification of previously described thermal decomposition procedure of an iron complex precursor. ${ }^{18}$ SPION were then coated with dimercaptosuccinic acid (DMSA) by a ligand exchange process $^{19,20}$ in order to disperse them in aqueous media. The SPION core size $(18 \mathrm{~nm}$, standard deviation $2 \mathrm{~nm})$ was determined by transmission electron microscopy while their hydrodynamic diameter in water dispersions $(65 \mathrm{~nm}$, Polydispersity index $=0.18$ ) was measured through dynamic light scattering. The colloidal suspension stability of our DMSA coated SPION dispersed in water was extremely high. No SPION sedimentation was observed for months or even after applying $H_{\mathrm{AC}}$ for hours leading to high reproducibility of the calorimetry measurements. Thermal measurements were performed in a home-made set-up of reduced volume (up to $40 \mu \mathrm{l})$. For such reduced volume, we can assume a constant temperature into the solution. The sample holder is a glass flask with a vacuum shield covered by a polystyrene stopper where an upper aperture allows to introduce the temperature 
probe. This aperture is responsible for the thermal exchange of the sample with the surroundings (lab environment) via convection mechanisms. $H_{\mathrm{AC}}$ up to $250 \mathrm{kHz}$ and $50 \mathrm{mT}$ are generated by a home-made air-cooled ferrite core, coiled with Litz wires, which is part of a LCR resonant circuit. The SPION temperature was measured with a commercial optical fibre probe TS2/2 connected to a FOTEMP2-16 two-channel signal conditioner from Optocon AG with an experimental error of $\pm 0.2^{\circ} \mathrm{C}$. For the present study, calorimetry measurements have been performed on a $35 \mu \mathrm{l}$ volume of SPION dispersed in distilled water with an iron concentration of $10 \mathrm{~g} / \mathrm{l}$. SPION were subjected to the same given $H_{\mathrm{AC}}$ conditions ( $78 \mathrm{kHz}$ and $25 \mathrm{mT}$ during $8 \mathrm{~min}$ ) for different initial temperatures $\left(T_{\mathrm{i}}\right)$ and $T_{\text {eq }}$ in the range from $10{ }^{\circ} \mathrm{C}$ to $30^{\circ} \mathrm{C}$.

Figure 1 compares calorimetry measurements recorded at slightly different $T_{\mathrm{eq}}$. At first glance, a similar behaviour of temperature variation is observed: a sudden SPION temperature increase (thin line) when $H_{\mathrm{AC}}$ is on due to the magnetically induced heating of SPION followed by a progressive temperature saturation. Finally, SPION temperature decays (thick line) when $H_{\mathrm{AC}}$ is turned off due to SPION heat losses into the surrounding. Under non-adiabatic conditions, the saturation temperature $\left(T_{\text {sat }}\right)$ is related to a thermal equilibrium reached when the SPION heat losses compensate the magnetic heating power. Fig. 1(a) shows the temperature variation of two consecutive measurements when $H_{\mathrm{AC}}$ is turned on at $T_{\mathrm{i}}=T_{\mathrm{eq}, 1}=30.2^{\circ} \mathrm{C}$, i.e., under equilibrium conditions. Within experimental error, the different measurement trials show a reproducibility better than $99 \%$. In turn, Fig. 1(b) shows the SPION temperature variation when $H_{\mathrm{AC}}$ is turned on at different $T_{\mathrm{i}}$ for $T_{\mathrm{eq}, 2}=28.4^{\circ} \mathrm{C}$. Note that the measurements starting at $T_{\mathrm{i}}>T_{\text {eq, } 2}$ are performed under thermal non-equilibrium conditions. Thus, the temperature increment $(\Delta T)$ has similar values of $\approx 10^{\circ} \mathrm{C}$ under thermal equilibrium conditions (i.e., when $T_{\mathrm{i}}=T_{\mathrm{eq}}$ )), whilst

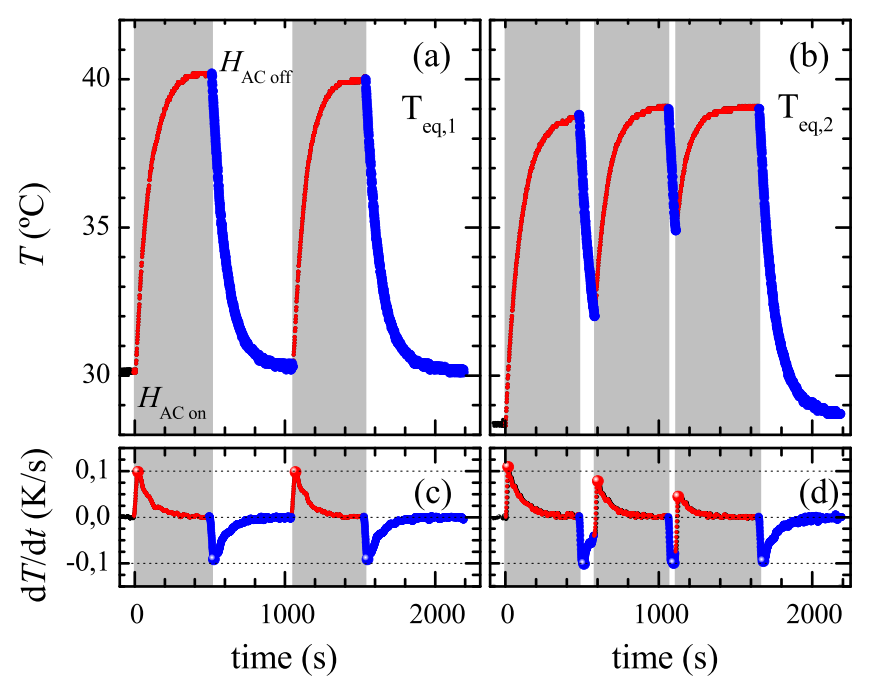

FIG. 1. Time evolution of SPION temperature when subjected to $H_{\mathrm{AC}}$ (78 kHz and $25 \mathrm{mT}$ ) (a) at $T_{\text {eq }, 1}=30.2^{\circ} \mathrm{C}$, (b) at $T_{\text {eq }, 2}=28.4^{\circ} \mathrm{C}$ for different $T_{\mathrm{i}}$ when applying $H_{\mathrm{AC}}$. (c) and (d) Numerical derivative curves of data shown in (a) and (b), respectively. Shadow and white time zones are exposed to $H_{\mathrm{AC}}$ on and off, respectively. Thin and thick lines correspond to temperature rise and decay. Solid circles in (c) and (d) indicate the maximun and minimun $\mathrm{d} T / \mathrm{d} t$ values.
$\Delta T<10^{\circ} \mathrm{C}$ when $T_{\mathrm{i}}>T_{\text {eq. }}$. As mentioned below, this will have also relevant implications on the value of $\mathrm{d} T /\left.\mathrm{d} t\right|_{\mathrm{t}=0}$.

A first quantitative SAR analysis can be derived when numerically differentiating the temperature curves (see Figs. 1(c) and 1(d)). In general, SAR value is determined from the temperature increase at initial times immediately after turning $H_{\mathrm{AC}}$ on, i.e., $\mathrm{d} T /\left.\mathrm{d} t\right|_{t=0}$. In practice, the conventional procedure currently employed for determining SAR values is based on quantifying the maximum value of $\mathrm{d} T / \mathrm{d} t(t)$ at initial times, i.e., $\mathrm{d} T /\left.\mathrm{d} t\right|_{\max }$.

Fig. 1(c) shows the derivative of the temperature variation plotted in Fig. 1(a). One can observe identical positive and negative $\mathrm{d} T / \mathrm{d} t$ behaviour under thermal equilibrium conditions at $T_{\text {eq, } 1}$. The maximum and minimum values of $\mathrm{d} T / \mathrm{d} t$ have an identical absolute value of $|\mathrm{d} T / \mathrm{d} t|_{\max / \min }$ $=0.11 \mathrm{~K} / \mathrm{s}$ (see solid circles in Fig. 1(c)). Contrary, a different $\mathrm{d} T / \mathrm{d} t$ behavior is observed under thermal nonequilibrium conditions (i.e., $T_{\mathrm{i}}>T_{\text {eq }}$ ). Fig. 1(d) shows the derivative curve of the temperature variation shown in Fig. 1(b). As one can see, while the negative part of $\mathrm{d} T / \mathrm{d} t$ reaches similar minimum values along the whole time span, the maximum values of $\mathrm{d} T / \mathrm{d} t$ decrease when increasing the difference between $T_{\mathrm{i}}$ and $T_{\mathrm{eq}}$. This fact stresses that SPION SAR values are properly defined only when calorimetry measurements are performed under thermal equilibrium, otherwise SAR values are underestimated.

In case of our calorimetry measurements, these equilibrium conditions are equally found when $H_{\mathrm{AC}}$ is turned on and off at temperatures $T_{\mathrm{eq}}$ and $T_{\text {sat }}$, respectively. In the first case, the SPION temperature variation at initial times immediately after applying $H_{\mathrm{AC}}$ is mainly influenced by the magnetically induced heating power of SPION and scarcely by heat transfer losses. ${ }^{14}$ In the second case, the SPION temperature variation is influenced by the vanishing of the magnetically induced heating power when turning $H_{\mathrm{AC}}$ off. In both cases, the heat transfer losses of SPION into the surrounding are the same. Hence, similar analysis than in case of turning $H_{\mathrm{AC}}$ on can be performed when $H_{\mathrm{AC}}$ is turned off, independently of the heat losses mechanisms involved. As shown in Figs. 1(c) and 1(d), identical values of $\mathrm{d} T /\left.\mathrm{d} t\right|_{\min }=$ $-0.11 \mathrm{~K} / \mathrm{s}$ are observed. Thus, SAR can be equally determined under thermal equilibrium conditions by considering the initial rise or decay slope immediately after turning $H_{\mathrm{AC}}$ on or off, respectively, leading to the value of $46 \mathrm{~W} / \mathrm{g}$.

On the other hand, the conventional procedure involves some accuracy drawbacks. First, a linear fitting of the temperature slope immediately after applying $H_{\mathrm{AC}}$ is commonly established considering the same time interval and number of points (in our case $20 \mathrm{~s}$ and 20 points) and the experimental error of the temperature measurement $\left( \pm 0.2^{\circ} \mathrm{C}\right)$. In the precedent analysis, under equilibrium conditions the slope reads $0.11 \pm 0.01 \mathrm{~K} / \mathrm{s}$, leading to $\mathrm{SAR}=46 \pm 4 \mathrm{~W} / \mathrm{g}(10 \%$ relative inaccuracy). However, the absolute error is independent of the slope value, which may results in huge relative inaccuracies for small SAR values. Second, the heat transfer losses at initial times after applying $H_{\mathrm{AC}}$ lead to further inaccuracies. Both uncertainty sources can be properly considered when evaluating the heat loss mechanisms along the whole temperature variation curve, instead of the initial times only. 
In order to improve the accuracy on determining the SPION SAR values under non-adiabatic conditions, we propose a simple model based on linear heat losses of SPION. We assume that convection mechanism is the only heat transfer of SPION into the surrounding. The reduced SPION volume and the thermal insulation of the glass flask lead to negligible conduction and radiation losses. Later on, experimental data will confirm this assumption. Thus, from a simple convection rate equation, ${ }^{15}$ we can derive solutions for $T_{\text {rise }}$ and $T_{\text {decay }}$ whose expressions read:

$$
\begin{aligned}
T_{\text {rise }}(t) & =T_{\text {eq }}+\Delta T\left(1-\mathrm{e}^{-a\left(t-t_{\text {on }}\right)}\right) \\
T_{\text {decay }}(t) & =T_{\text {sat }}-\Delta T\left(1-\mathrm{e}^{-a\left(t-t_{\text {off }}\right)}\right),
\end{aligned}
$$

where $\Delta T=T_{\text {sat }}-T_{\text {eq }}, a=k / C, k$ is the heat exchange of SPION into the surrounding, $C$ is the specific heat of the sample, $t_{\mathrm{on}}$ and $t_{\mathrm{off}}$ are the times when $H_{\mathrm{AC}}$ is turned on and off, respectively. Notice that $T_{\text {rise }}$ and $T_{\text {decay }}$ have a similar but inverted time dependence. Our model has a single fitting parameter, namely $a$ since $T_{\text {eq }}$ and $T_{\text {sat }}$, and consequently $\Delta T$, are determined from experimental data. Hence, the numerical value of $\mathrm{d} T /\left.\mathrm{d} t\right|_{t=t_{\mathrm{on}} \text { off }}=\Delta T \cdot a$. The uncertainty of the numerical value of $\mathrm{d} T /\left.\mathrm{d} t\right|_{t=t_{\text {on } / \text { fff }}}$ can be now determined by Gaussian error propagation, which is a valid method for providing an interval of confidence to the numerical results derived from Eqs. (1). Now, SAR accuracy depends on the values and inaccuracies of $\Delta T$ and $a$, and is tested in larger number of points and time interval than the conventional procedure mentioned above.

Figure 2 compares the experimental data plotted at Figs. 1(a) and 1(b) with the fitting curves obtained from Eqs. (1) in order to calculate the temperature increase and decay when $H_{\mathrm{AC}}$ is turned on and off, respectively. The model is in good agreement with experimental results, being able to completely reproduce calorimetry measurements starting at $T_{\mathrm{i}}>T_{\text {eq }}$. The fitting parameter value employed is $a=0.0110 \pm 0.0005 \mathrm{~s}^{-1}$. Note that now the relative SAR uncertainties are smaller than 5\% what represents a significant increase of accuracy with respect to the conventional procedure (i.e., $\mathrm{SAR}=46 \pm 2 \mathrm{~W} / \mathrm{g}$ ).

In order to verify the validity of our assumptions on heat loss linearity, we perform experiments at $T_{\mathrm{eq}, 3}=10.0^{\circ} \mathrm{C}$,

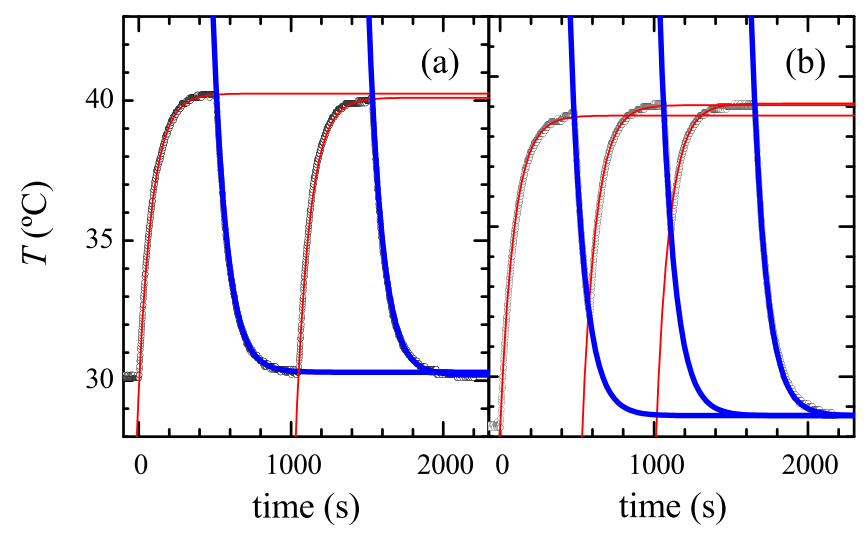

FIG. 2. Comparison of simulations and experimental data at (a) $T_{\text {eq }, 1}=30.2^{\circ} \mathrm{C}$ and (b) $T_{\text {eq }, 2}=28.4{ }^{\circ} \mathrm{C}$. Circles correspond to experimental data, thin and thick lines correspond to numerical simulations obtained from Eqs. (1), with the fitting parameter $a=0.0110 \pm 0.0005 \mathrm{~s}^{-1}$.

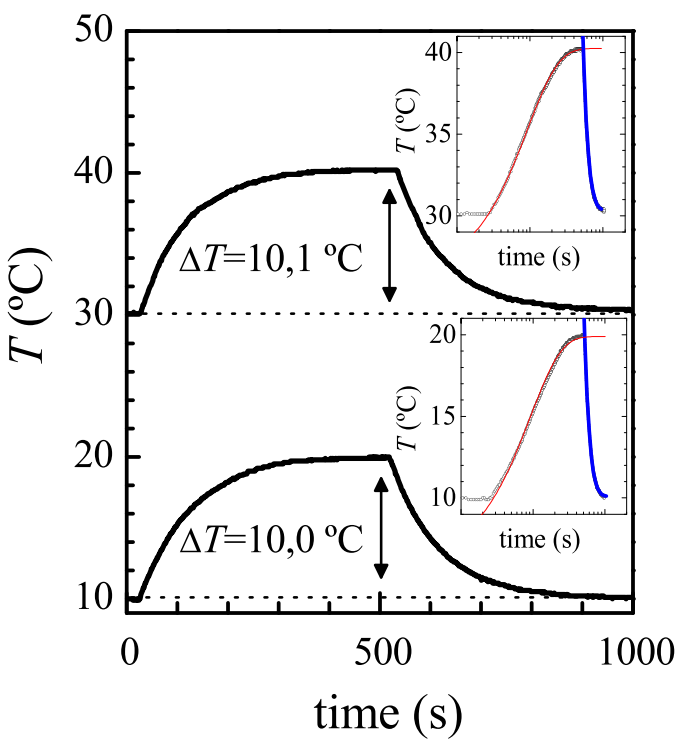

FIG. 3. Time evolutions of SPION temperature at $T_{\text {eq, } 1}=30.2^{\circ} \mathrm{C}$ and $T_{\text {eq }, 3}$ $=10.0^{\circ} \mathrm{C}$ when subjected to $H_{\mathrm{AC}}(78 \mathrm{kHz}$ and $25 \mathrm{mT})$. Insets: Comparison of numerical simulations and experimental data. Circles correspond to experimental data, thin and thick lines correspond to the numerical simulations obtained by using Eqs. (1), with the fitting parameter $a=0.0110 \pm 0.0005 \mathrm{~s}^{-1}$.

i.e., a temperature $20^{\circ} \mathrm{C}$ lower than previously. Figure 3 compares calorimetry measurements performed at $T_{\text {eq }, 1}=30.2^{\circ} \mathrm{C}$ and $T_{\text {eq }, 3}=10.0^{\circ} \mathrm{C}$. Identical temperature behaviour with a similar $\Delta T$ values than at higher temperatures $T_{\text {eq }}$ are observed at both equilibrium temperatures. This indicates that the SPION thermal losses by radiation mechanism (proportional to $T^{4}-T_{\text {eq }}^{4}$ ) are negligible in the studied temperature range (from $10^{\circ} \mathrm{C}$ to $30^{\circ} \mathrm{C}$ ). This finding justifies the assumption of linear heat losses. Hence, the only heat transfer losses in our experimental systems may correspond to convection mechanisms as initially assumed. In fact, as shown in insets of Fig. 3, model calculations using the same fitting parameter value highly agree with the experimental results leading to similar SAR values within the studied temperature range.

In summary, we have studied the temperature variation of SPION subjected to $H_{\mathrm{AC}}$ under non-adiabatic conditions. Our experimental results show similar temperature variation curves for given $H_{\mathrm{AC}}$ conditions at different $T_{\mathrm{eq}}$ ranging from $10^{\circ} \mathrm{C}$ to $30^{\circ} \mathrm{C}$. The use of reduced volumes of SPION water dispersions allows to precisely simulate calorimetry measurements by a simple model that considers the linearity of SPION heat losses into the surrounding. The control and the quantification of heat losses lead to higher accuracy in determining SAR values, for example, to disentangle the influence of the intrinsic SPION parameters (i.e., particle size or concentration) as well as extrinsic ones (i.e., $H_{\mathrm{AC}}$ conditions) on the SAR values. The proposed thermal description will remarkably improve the accuracy on the quantification of the thermal transfer induced by SPION for optimizing the efficiency of the magnetic heating.

This work has been partially supported by EU-FP7 MULTIFUN Project (No. 262943), by Spanish Ministry of Economy and Competitiveness (MAT2010-21822-C02-01 and CSD2007-00010) and NANOBIOMAGNET Project (S2009/MAT-1726) funded by Comunidad de Madrid. F.J.T 
$062413-4$

Teran et al.

Appl. Phys. Lett. 101, 062413 (2012)

and A.B. acknowledge financial support from Ramon y Cajal subprogram (RYC-2011-09617 and RYC-2007-01727, respectively).

${ }^{1}$ A. Villanueva, M. Cañete, A. G. Roca, M. Calero, S. Veintemillas-Verdaguer, C. J. Serna, M. P. Morales, and R. Miranda, Nanotechnology 20, 115103 (2009).

${ }^{2}$ A. G. Roca, R. Costo, A. F. Rebolledo, S. Veintemillas-Verdaguer, P. Tartaj, T. González-Carreño, M. P. Morales, and C. J. Serna, J. Phys. D: Appl. Phys. 42, 224002 (2009).

${ }^{3}$ J. M. Idée M. Port, I. Raynal, M. Schaefer, B. Bonnemain, P. Prigent, P. Robert, C. Robic, and C. Corot, Nanomaterials for Medical Diagnosis and Therapy, Nanotechnologies for Life Sciences, Vol. 10 (Wiley, 2007), p. 51.

${ }^{4}$ R. Mejías, S. Pérez-Yagüe, L. Gutiérrez, L. I. Cabrera, R. Spada, P. Acedo, C. J. Serna, F. J. Lázaro, A. Villanueva, M. Puerto Morales, and D. F. Barber, Biomaterials 32, 2938 (2011).

${ }^{5}$ A. Jordan, P. Wust, H. Fählin, W. John, A. Hinz, and R. Felix, Int. J. Hyperthermia 9, 51 (1993).

${ }^{6}$ R. E. Rosensweig, J. Magn. Magn. Mater. 252, 370 (2002).

${ }^{7}$ B. Mehdaoui, A. Meffre, J. Carrey, S. Lachaize, L. M. Lacroix, M. Gougeon, B. Chaudret, and M. Respaud, Adv. Funct. Mater. 21, 4573 (2011).

${ }^{8}$ M. Jeun, S. Lee, J. K. Kang, A. Tomitaka, K. W. Kang, Y. I. Kim, Y. Takemura, K. W. Chung, J. Kwak, and S. Bae, Appl. Phys. Lett. 100, 092406 (2012).

${ }^{9}$ Y. Piñeiro-Redondo, M. Bañobre-López, I. Pardiñas-Blanco, G. Goya, M. A. López-Quintela, and J. Rivas, Nano. Res. Lett. 6, 383 (2011).
${ }^{10}$ D. Serantes, D. Baldomir, C. Martinez-Boubeta, K. Simeonidis, M. Angelakeris, E. Natividad, M. Castro, A. Mediano, D.-X. Chen, A. Sanchez, Li. Balcells, and B. Martínez, J. Appl. Phys. 108, 073918 (2010).

${ }^{11}$ A. S. Eggeman, S. A. Majetich, D. Farrell, and Q. A. Pankhurst, IEEE Trans. Magn. 43, 2451 (2007).

${ }^{12}$ S. Huang, S. Y. Wang, A. Gupta, D. A. Borca-Tasciuc, and S. J. Salon, Meas. Sci. Technol. 23, 035701 (2012).

${ }^{13}$ P. Guardia, R. Di Corato, L. Lartigue, C. Wilhelm, A. Espinosa, M. Garcia-Hernandez, F. Gazeau, L. Manna, and T. Pellegrino, ACS Nano 6(4), 3080 (2012).

${ }^{14}$ E. Natividad, M. Castro, and A. Mediano, Appl. Phys. Lett. 92, 093116 (2008); E. Natividad, M. Castro, and A. Mediano, J. Magn. Magn. Mater. 321, 1497 (2009).

${ }^{15}$ F. P. Incropera, D. P. DeWitt, T. L. Bergman, and A. S. Lavine, Introduction to Heat Transfer, 5th ed. (Wiley, 2007).

${ }^{16}$ I. Marcos-Campos, L. Asín, T. E. Torres, C. Marquina, A. Tres, M. R. Ibarra, and G. F. Goya, Nanotechnology 22, 205101 (2011).

${ }^{17}$ C. L. Dennis, A. J. Jackson, J. A. Borchers, P. J. Hoopes, R. Strawbridge, A. R. Foreman, J. van Lierop, C. Grüttner, and R. Ivkov, Nanotechnology 20, 395103 (2009).

${ }^{18}$ J. Park, K. An, Y. Hwang, J.-G. Park, H.-J. Noh, J.-Y. Kim, J.-H. Park, N.-M. Hwang, and T. Hyeon, Nature Mater. 3, 891 (2004).

${ }^{19}$ Y.-W. Jun, Y.-M. Huh, J.-S. Choi, J.-H. Lee, H.-T. Song, S. Kim, S. Yoon, K.-S. Kim, J.-S. Shin, J.-S. Suh, and J. Cheon, J. Am. Chem. Soc. 127, 5732 (2005).

${ }^{20}$ Y.-M. Huh, Y. Jun, H.-T. Song, S. Kim, J. Choi, J.-H. Lee, S. Yoon, K. Kim, J.-S. Shin, J.-S. Suh, and J. Cheon, J. Am. Chem. Soc. 127, 12387 (2005). 
Applied Physics Letters is copyrighted by the American Institute of Physics (AIP). Redistribution of journal material is subject to the AIP online journal license and/or AIP copyright. For more information, see http://ojps.aip.org/aplo/aplcr.jsp 